\title{
Wie hoch ist die Rendite privater Forschung?
}

\author{
Flurina Mark
}

\section{Relevanz}

Mit privater Forschung und Entwicklung bauen die Unternehmen ihre Wettbewerbsfähigkeit aus und steigern Innovation und Wachstum der Gesamtwirtschaft. Dabei lernen und profitieren sie von den F\&E-Erfolgen und Erkenntnissen ihrer Mitbewerber mit ähnlicher technologischer Ausrichtung. Wenn dagegen die Konkurrenten auf den Absatzmärkten aufrüsten und Marktanteile besetzen, ist es für die Absatzchancen und den eigenen F\&E-Ertrag weniger gut bestellt. Die Forschung zeigt jedoch, dass die Vorteile privater F\&E für andere Unternehmen deutlich überwiegen. Daher ist die F\&E-Rendite für die gesamte Wirtschaft viel höher als die private Rendite eines einzelnen Unternehmens. Wenn die Rendite gering ist, investiert man weniger. Die Gesellschaft kann viel gewinnen, wenn sie private F\&E erleichtert und unterstützt.

\section{Quelle}

Brian Lucking, Nicholas Bloom und John Van Reenen (2018), Have R\&D Spillovers Changed? NBER Working Paper No. 24622.

Viele Unternehmen betreiben Forschung und Entwicklung (F\&E). Damit bringen sie die Innovation und das Wachstum der Wirtschaft voran. Forschung und Entwicklung nützen in erster Linie dem Unternehmen selbst, welches in diese Aktivitäten investiert. Zudem beeinflussen sie indirekt auch die anderen Unternehmen.

F. Mark $(\bowtie)$

Universität St.Gallen, St.Gallen, Schweiz

E-Mail: flurina.mark@student.unisg.ch 
Einerseits kann F\&E die Produktivität von Unternehmen steigern, welche ähnliche Technologien verwenden und daher über Prozessinnovationen und technologische Verbesserungen lernen und davon profitieren. Andererseits leiden Wettbewerber, die ähnliche Güter anbieten. Denn innovativere Produkte verringern typischerweise die Nachfrage nach Konkurrenzprodukten. Wie lassen sich diese zwei gegenläufigen Effekte empirisch unterscheiden und quantifizieren?

Die Ökonomen Brian Lucking, Nicholas Bloom und John Van Reenen von den Universitäten Stanford und MIT untersuchen, wie sich F\&E auf andere Unternehmen auswirkt („Spillover-Effekte“). Dazu verwenden sie Daten zu Patenten, finanziellen Kennzahlen und Performance US-amerikanischer Unternehmen zwischen 1980 und 2015. Im Vergleich zu früheren Studien erstreckt sich ihr Datensatz über einen längeren Zeitraum und umfasst auch neuere Patente.

Wie kann man die positiven und negativen Spillover-Effekte in den Daten erfassen? Dabei spielt die Nähe von Unternehmen entweder im Sinne ähnlicher Technologien oder vergleichbarer Absatzmärkte ihrer Produkte eine wichtige Rolle. So gelten zwei Unternehmen, welche über viele Patente in denselben Technologiebereichen verfügen oder hohe Umsätze in den gleichen Branchen erzielen, als nahe. Im ersten Fall ist ein positiver, im zweiten Fall aber ein negativer Spillover-Effekt der F\&E plausibel. Die so beschriebene Nähe misst, wie stark ein Unternehmen gegenüber den F\&E-Aktivitäten anderer Unternehmen exponiert ist. Die Interdependenzen hängen jedoch nicht allein von der Nähe ab, sondern auch vom Umfang der Forschungsaktivitäten Dritter. Je näher und forschungsintensiver andere Unternehmen sind, desto stärker fallen die positiven und negativen Spillover-Effekte aus.

Die Wissenschaftler analysieren die Auswirkungen von F\&E auf verschieden Unternehmenskennzahlen wie Marktwert, Anzahl Patente, und F\&E-Ausgaben. Die zentrale Hypothese ist, dass der Marktwert eines Unternehmens mit den F\&E-Aktivitäten anderer, technogisch vergleichbarer Unternehmen steigt. Die Anzahl der Patente und die Produktivität sollten sich erhöhen. Intensivere Forschungsanstrengungen der Konkurrenten, welche ihre Marktanteile ausbauen wollen, werden sich dagegen eher negativ auf die betroffenen Unternehmen auswirken. Wie sich positive oder negative Spillover-Effekte auf die F\&E-Ausgaben eines Unternehmens auswirken, ist also a priori nicht eindeutig.

Die empirischen Schätzungen zeigen, dass die Spillover-Effekte den Marktwert signifikant beeinflussen. Nehmen die Forschungsaktivitäten naher Unternehmen mit ähnlicher Technologie um $10 \%$ zu, so erhöht sich der Marktwert eines Unternehmens um 32,4\%. Ein vergleichbarer Anstieg der F\&E bei den unmittelbaren Konkurrenten auf den Absatzmärkten verringert dagegen den Marktwert um 8,6\%. Zudem schätzen John Van Reenen und seine Ko-Autoren einen Anstieg der Patentzahlen, wenn Unternehmen mit ähnlicher Technologie sehr forschungsintensiv sind. Weiter nimmt die Produktivität um 23,1 \% zu, wenn technologisch nahe Unternehmen ihre F\&E- 
Aktivitäten um $10 \%$ steigern. Ihre Ergebnisse zeigen aber auch, dass forschungsintensivere Konkurrenten, die um dieselben Marktanteile kämpfen, den Nutzen der eigenen F\&E tendenziell schmälern und die Zahl der Patente verringern.

Schliesslich erhöhen beide Spillover-Effekte die Forschungsausgaben eines Unternehmens, zum einen, weil sie von den Erfahrungen und Erkenntnissen technologisch naher Mitbewerber profitieren, zum anderen aber auch, um sich gegen die Konkurrenten auf den Absatzmärkten zu wehren. F\&E der anderen Unternehmen verstärkt daher den Anreiz, selbst mehr in Forschung und Entwicklung zu investieren.

Steigen die Forschungsanstrengungen anderer Unternehmen mit einer ähnlichen Technologie um $10 \%$, vergrössert sich der Marktwert um $32.4 \%$. Nehmen die F\&EAktivitäten der Produktmarktkonkurrenten um $10 \%$ zu, sinkt der Marktwert um $8.6 \%$.

In einem nächsten Schritt dokumentieren die Forscher, dass sich zwischen 1980 und 2015 die positiven und negativen Nebenwirkungen privater F\&E insgesamt nur wenig verändert haben. Während des Dotcom-Booms zwischen 1995 und 2005 waren die positiven Spillover-Effekte auf den Marktwert um $48 \%$ grösser und die negativen um $38 \%$ niedriger. Dies spiegelt möglicherweise den Enthusiasmus der Investoren für forschungsintensive Unternehmen zu jener Zeit wider.

Die soziale Ertragsrate zusätzlicher F\&E ist rund viermal so hoch wie die private Rendite. Weil die Unternehmen nicht alle gesellschaftlich relevanten Erträge der Innovation berücksichtigen, fallen die privaten $\mathrm{F} \& \mathrm{E}-$ Investitionen zu gering aus.

Schliesslich schätzen die Wissenschaftler die sozialen und privaten Renditen zusätzlicher F\&E-Investitionen. Der private Ertrag misst die Veränderung der eigenen Wertschöpfung aufgrund höherer F\&E-Aktivität jenes Unternehmens. Hingegen misst der soziale Ertrag die Veränderung der gesamten Wertschöpfung aller Unternehmen gemeinsam und berücksichtigt damit die positiven und negativen Spillover-Effekte der privaten F\&E. John Van Reenen und seine Ko-Autoren schätzen den sozialen Ertrag auf 57,7 \% und den privaten auf 13,6 \%. Die sozialen Ertragsraten waren somit rund viermal so hoch wie private. Dieser Unterschied fiel stärker aus als in früheren Studien mit einem kürzeren Beobachtungszeitraum. Diese schätzten eine etwa dreimal höhere soziale Rendite. Der zunehmende Unterschied zwischen der sozialen und privaten Ertragsrate war überwiegend einem kleineren privaten Grenzertrag der F\&E zuzuschreiben. Dass der soziale Ertrag den privaten übersteigt, weist auf ineffizient niedrige F\&E-Aktivitäten von Unternehmen hin. Sie berücksichtigen bei ihrer eigenen F\&E-Entscheidung nicht, dass auch andere Unternehmen davon profitieren würden. Aus gesellschaftlicher Sicht wäre daher eine Ausdehnung privater F\&E-Investitionen wünschenswert. 


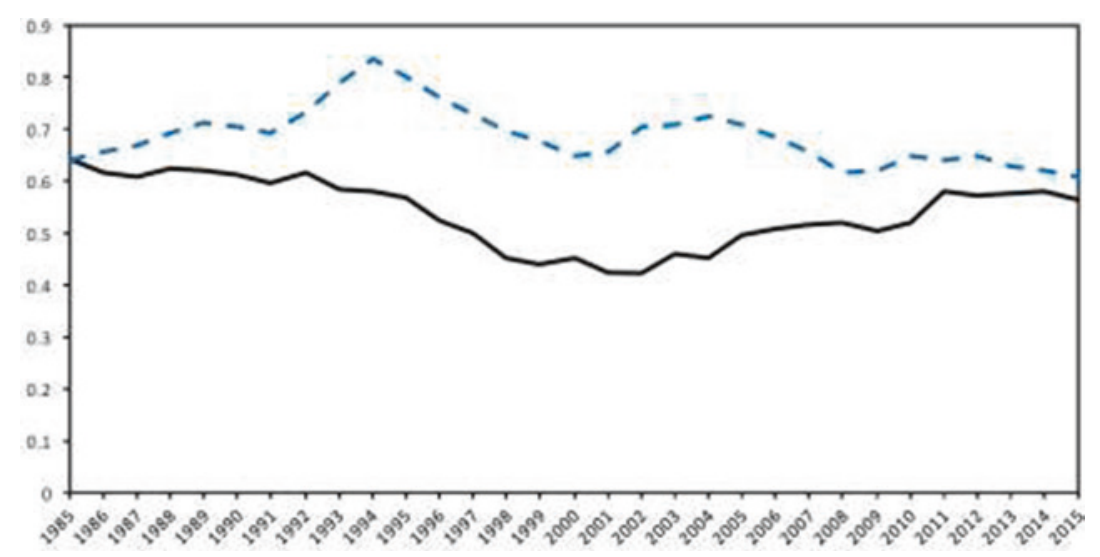

Abb. 1 Die soziale Ertragsrate von F\&E-Investitionen. (Quelle: Lucking et al. 2018)

Abschliessend untersuchten die Forscher die Veränderung der sozialen Ertragsrate über die gesamte Zeitperiode von 1985 bis 2015 (siehe Abb. 1). Sie berechneten diese Grösse sowohl für die Unternehmen in der Stichprobe (schwarze Linie) als auch für alle US-amerikanischen Unternehmen anhand aggregierter Daten (blaue gestrichelte Linie). Letzteres sollte einer Verzerrung vorbeugen. Denn die beobachteten Unternehmen waren vergleichsweise forschungsintensiv und daher möglicherweise nicht repräsentativ für die gesamte Unternehmenslandschaft der USA. Die Wissenschaftler stellen jedoch fest, dass die soziale Rendite unabhängig davon keine massiven Schwankungen verzeichnete und sich relativ stabil verhielt.

Open Access Dieses Kapitel wird unter der Creative Commons Namensnennung 4.0 International Lizenz (http://creativecommons.org/licenses/by/4.0/deed.de) veröffentlicht, welche die Nutzung, Vervielfältigung, Bearbeitung, Verbreitung und Wiedergabe in jeglichem Medium und Format erlaubt, sofern Sie den/die ursprünglichen Autor(en) und die Quelle ordnungsgemäß nennen, einen Link zur Creative Commons Lizenz beifügen und angeben, ob Änderungen vorgenommen wurden.

Die in diesem Kapitel enthaltenen Bilder und sonstiges Drittmaterial unterliegen ebenfalls der genannten Creative Commons Lizenz, sofern sich aus der Abbildungslegende nichts anderes ergibt. Sofern das betreffende Material nicht unter der genannten Creative Commons Lizenz steht und die betreffende Handlung nicht nach gesetzlichen Vorschriften erlaubt ist, ist für die oben aufgeführten Weiterverwendungen des Materials die Einwilligung des jeweiligen Rechteinhabers einzuholen.

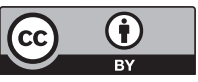

